\title{
Alpha-2 Adrenergic Modulation of Prefrontal Cortical Neuronal Activity Related to Spatial Working Memory in Monkeys
}

\author{
Bao-Ming Li, Ph.D., Zheng-Mei Mao, B.M., Min Wang, B.S., and Zhen-Tong Mei, Ph.D.
}

The effects of systemically administered or iontophoretically applied clonidine (alpha-2 adrenergic agonist) and iontophoretically applied yohimbine (alpha-2 adrenergic antagonist) were examined on prefrontal cortical (PFC) neurons related to spatial working memory (SWM). Systemically administered clonidine $(0.04 \mathrm{mg} / \mathrm{kg})$ enhanced SWM-related PFC neuronal activity by $32.5 \pm 14.5 \%$, (mean $\pm S D ; \mathrm{n}=25$ neurons). The facilitatory effect of clonidine was antagonized by iontophoretically applied yohimbine. Iontophoretically applied clonidine enhanced SWM-related PFC neuronal activity by $38.2 \pm 18.6 \%$, ( $\mathrm{n}=13$ neurons), whereas similarly applied yohimbine suppressed it by $34.4 \pm 17.8 \%$ ( $\mathrm{n}=28$ neurons). These results indicate that: a) systemically administered clonidine can facilitate SWM-related PFC neuronal activity through actions at alpha-2 adrenoceptors in the PFC; and b) conversely, blockade by yohimbine of alpha-2 adrenoceptors in the PFC suppresses SWM-related neuronal activity. The present study provides neurophysiological evidence that alpha-2 adrenoceptors in the PFC are involved in the cellular mechanisms underlying working memory.

[Neuropsychopharmacology 21:601-610, 1999] (C) 1999 American College of Neuropsychopharmacology. Published by Elsevier Science Inc.
KEY WORDS: Clonidine; Yohimbine; Alpha-2 adrenoceptor; Working memory; Neuronal activity; Prefrontal cortex; Monkey

The prefrontal cortex (PFC) plays a critical role in regulating spatial working memory (SWM) (Fuster et al. 1982; Funahashi et al. 1989; di Pellegrino and Wise 1993; Wilson et al. 1993; Miller et al. 1996; Rao et al. 1997. Goldman-Rakic 1987, 1996; Funahashi and Kubota 1994). Many neurons in the PFC exhibit "memory fields", increasing their firing rate during the delay period following the presentation of a specific spatial cue (Goldman-Rakic 1987; Funahashi et al. 1989). This activ-

From the Key Laboratory of Neurobiology, Shanghai Institute of Physiology, Chinese Academy of Sciences, Shanghai 200031, People's Republic of China.

Address correspondence to: Bao-Ming Li, Ph.D., Key Laboratory of Neurobiology, Shanghai Institute of Physiology, Chinese Academy of Sciences, 320 Yue-Yang Road, Shanghai 200031, P. R. China.

Received December 14, 1998; revised May 26, 1999; accepted June 9, 1999. ity during the delay period, when the cue is no longer present, is generally considered to be the cellular manifestation of working memory (ibid).

Behavioral and pharmacological studies have established that alpha-2 adrenoceptors in the PFC are linked with SWM (Arnsten et al. 1996). For example, systemically administered alpha-2 agonists improve SWM in aged monkeys with naturally occurring degeneration of prefrontal norepinephrine (NE) fibers (Arnsten and Goldman-Rakic 1985; Arnsten et al. 1988; Arnsten and Leslie 1991; Rama et al. 1996). Improvement has also been observed in young monkeys with 6-OHDA-induced depletion of prefrontal catecholamines (Brozoski et al. 1979; Arnsten and Goldman-Rakic 1985) or with reserpine-induced global loss of catecholamines (Cai et al. 1993). Indeed, the potency of the systemically administered alpha- 2 agonists is directly related to the degree of NE depletion, consistent with actions at postsynaptic alpha-2 adrenoceptors in the PFC (Arnsten and Goldman-Rakic 1985; Cai et al. 1993). 
Furthermore, the memory-improving effect of clonidine is no longer evident after the PFC is ablated (Arnsten and Goldman-Rakic 1985; Arnsten and van Dyck 1997). Finally, alpha-2 agonists and antagonists can alter working memory performance when infused directly into the PFC. Infusion of the alpha-2 agonist, medetomidine, into the PFC of aged rats improved delayed alternation performance (Tanila et al. 1996), whereas blockade of alpha-2 adrenoceptors in the PFC by local infusion of the antagonist, yohimbine, impairs performance of SWM in young adult monkeys ( $\mathrm{Li}$ and Mei 1994a).

Most recently, higher doses of clonidine (0.02-0.1 $\mathrm{mg} / \mathrm{kg}$ ) have been shown to improve working memory performance in normal, young adult monkeys (see accompanying paper by Franowicz and Arnsten 1999, this issue). However, it is unknown if systemically-administered alpha- 2 agonists modulate PFC neuronal activity related to SWM and whether stimulation or blockade of alpha-2 adrenoceptors in the PFC has an influence on SWM-related neuronal activity. This question is of direct clinical importance, as alpha-2 agonists are now being developed and used for PFC cognitive disorders such as Attention Deficits Hyperactivity Disorder (Hunt et al. 1985, 1995; Chappell et al. 1995; Horrigan and Barnhill 1995; Arnsten et al. 1996). In the present study, we investigated the effects of systemically administered and/or iontophoretically applied alpha-2 agonist (clonidine) and antagonist (yohimbine) on SWM-related PFC neuronal activity. A preliminary result of this study was reported at the 3rd Congress of the Federation of Asian and Oceanian Physiological Societies (FAOPS) (Li and Mei 1994b).

\section{METHODS}

\section{Subjects}

Two female monkeys (Macaca mulatta, 4-6 years old, weighing $4.0-4.5 \mathrm{~kg}$ ) were used in the present study and were cared for in accordance with the Guiding Principles in the Care and Use of Laboratory Animals issued by the National Institutes of Health, USA (1985) and the Guide for the Care and Use of Laboratory Primates issued by the Primate Research Institute of Kyoto University, Japan (1986).

\section{Behavioral Task}

The monkeys were trained on a delayed-response task as described previously ( $\mathrm{Li}$ and Mei 1994a). In brief, the monkeys used their right hand to press a lever to initiate each trial, and sustained the lever press for the task to continue. After a $0.5 \mathrm{sec}$ waiting period, a left or right light-emitting diode (LED; $5 \mathrm{~mm}$ in length and 2 $\mathrm{mm}$ in width; 7.0 degrees in visual angle), mounted on a left or right button switch, was turned on for $0.5 \mathrm{sec}$ (cue). Then, there was a delay period of 1.0-3.0 sec. After the delay, a central LED (4 mm in diameter), located at the central position between the left and right LEDs, was turned on for $1.5 \mathrm{sec}$ (choice period). During the choice period the monkeys were required to release the lever and then press the cued button switch.

After a correct choice, the monkeys were given a drop of water $(0.2-0.3 \mathrm{ml})$. Left-cue and right-cue trials were randomly presented. Horizontal EOGs were monitored by using two small round silver electrodes which were attached via electrode paste to the skin of the temporal side of the monkey's eyes. Figure 1 shows the arrangement of the delayed-response task.

\section{Surgery}

After the monkeys learned the task $(>85 \%$ correct rate of performance in five consecutive daily sessions), they were subjected to aseptic surgery using sodium pentobarbital $(35 \mathrm{mg} / \mathrm{kg})$ anesthesia, during which a stainless steel cylinder $(19 \mathrm{~mm}$ in diameter) was implanted over the left prefrontal cortex. The bone under the cylinder was removed. The principal sulcus could be identified visually after removal of the bone, which helped to determine roughly the cortical area for electrode penetration. The monkeys were re-trained for several sessions after they recovered from surgery.

\section{Neuronal Activity Recording}

A multi-barreled glass-coated tungsten microelectrode, which was developed by Li et al. (1990), was used for neuronal activity recording (through tungsten wire inside the central barrel of the electrode array) and drug iontophoresis (through the surrounding glass barrels of the electrode array which were filled with drug solutions). The advantage of this electrode array, if compared with a multi-barreled glass microelectrode or a multi-barreled glass-coated carbon-fiber microelectrode, is that it can penetrate across the monkeys' dura easily due to the support of the tungsten wire, and therefore pre-penetration anesthesia and microsurgery (for opening a hole in the dura and inserting the electrode into the cortex) are not necessary (Li et al. 1990). This improvement made iontophoresis experiments in the behaving monkey much easier. The delay period varied randomly between $1.0 \mathrm{sec}$ and $3.0 \mathrm{sec}$ during recording sessions and was fixed at $2.0 \mathrm{sec}$ only when delay-related activity was being sampled for constructing a histogram of the activity (10 trials for a histogram).

\section{Drug Administration}

Once a delay-related neuron, which showed change in discharge rate selectively during the delay of left- or 
A

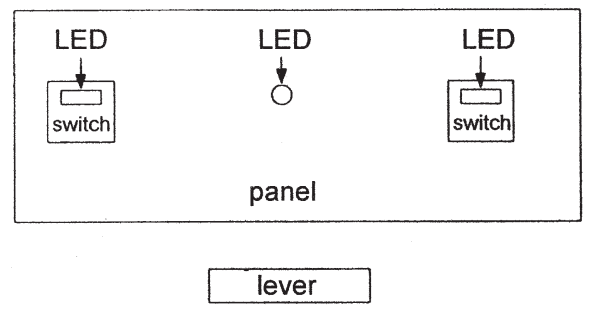

\section{B}

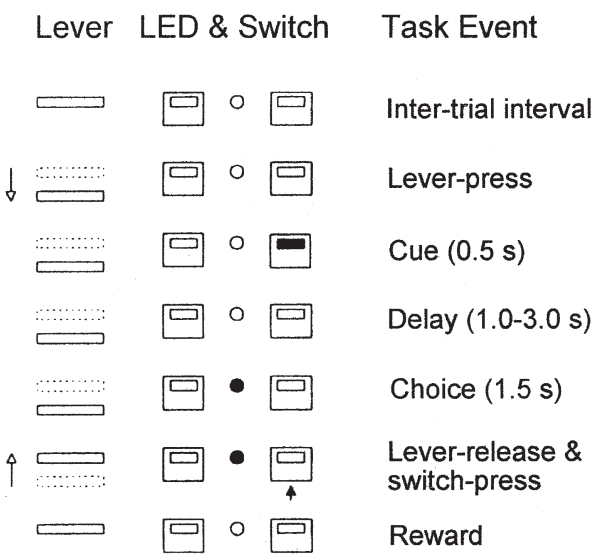

Figure 1. Arrangement of the delayed-response task. (A) A test panel was placed in front of the monkey. On the panel there were two button switches, on each of which a lightemitting diode (LED) was mounted. A central LED was located between the two button switches and a lever under the panel. (B) The monkey pressed the lever (indicated by the clear down arrow) and sustained the lever press to initiate the task. After a $0.5 \mathrm{sec}$ waiting period, the left or right LED (e.g., the right LED, as shown) was turned on for $0.5 \mathrm{sec}$ (cue). Then, there was a delay period of 1.0-3.0 sec. After the delay, the central LED was turned on for $1.5 \mathrm{sec}$ (choice period). During the choice period the monkey was required to release the lever (indicated by the clear up arrow) and then press the cued button switch (indicated by the solid up arrow). After a correct choice, the monkey was given a drop of water $(0.2-0.3 \mathrm{ml})$. For details see the text.

right-cue trials, appeared and its activity became stabilized, clonidine hydrochloride (Sigma Chemical Co., USA ) or yohimbine hydrochloride (Sigma Chemical Co.) was applied intramuscularly and/or iontophoretically (with a positive current of $10-80 \mathrm{nA}$, usually 50 $\mathrm{nA})$. For the application of clonidine hydrochloride, either intramuscular injection $(0.04 \mathrm{mg} / \mathrm{kg}$, dissolved in saline) or iontophoresis $(0.01 \mathrm{~mol} / \mathrm{L}$ in saline; $\mathrm{pH} 4.5)$ was used. The dose of intramuscularly injected clonidine was so chosen as it was one of the optimal doses for the improving effect of the drug on spatial working memory (see Arnsten and Goldman-Rakic 1985).
For the application of yohimbine hydrochloride, only iontophoresis $(0.01 \mathrm{~mol} / \mathrm{L}$ in saline; $\mathrm{pH} 4.5)$ was used. When iontophoresis was not being done, a negative current (10 nA) was continuously applied to prevent leakage of the drugs. As resistance exists from the site of the electrode tip to the ground, a voltage gradient will result when iontophoretic currents are caused to flow, which might affect neuronal firing. In order to prevent the appearance of the voltage gradient, we used current summing mode to balance iontophoretic currents. In the current summing mode, one of the iontophoresis modules (S-7061A, W-P Instruments, Inc., New Haven, CT, USA) and its associated barrel of the electrode array (filled with saline) was assigned the task of automatically absorbing the algebraic sum of all of the iontophoresis currents issuing from other barrels of the electrode array. Moreover, spike shape and discharge pattern of an examined neuron were carefully monitored before and during drug administration. This was especially important when clonidine was administered by intramascular injection, as the monkeys frequently moved during the injection which often caused the delay-related neuron to "die" or "disappear." At the end of each successful recording session, an electrolytic lesion (20 uA for 20-30 sec, positive current) was made for marking the site of electrode penetration.

\section{Data Analysis}

Neuronal activity during the waiting period was taken as baseline activity (all of the neurons reported in this paper showed no time-locked change in activity during the waiting period). Delay-related activity was judged to be sensitive to drug treatment if: 1) the value of the delay-related activity (spikes/sec) was significantly changed after (in the case of intramuscular injection) or during (in the case of iontophoresis) drug administration $(p<.05 ;$ Mann-Whitney's $U$-test); and 2) that of the baseline activity was unchanged $(p>.05$; Mann-Whitney's $U$-test).

\section{Histology}

After data collection was completed, each monkey was anesthetized with an overdose of pentobarbital sodium $(50 \mathrm{mg} / \mathrm{kg})$. A tungsten electrode was inserted into the cortex at the central position of the cylinder and a relatively strong positive current (60 nA for $60 \mathrm{sec}$ ) was passed through the electrode in order to mark the central position clearly as a reference site for re-construction of electrode penetration. Then, the monkey was perfused with saline $(500 \mathrm{ml})$ followed by $10 \%$ formalin solution $(500 \mathrm{ml})$ through the carotid artery. The left frontal lobe of each monkey was cut coronally into serial sections (100 $\mu \mathrm{m}$ in thickness) and the brain sections were stained with the Nissl method. The record- 
Table 1. Effects of Clonidine and Yohimbine and DelayRelated Prefrontal Cortical Neurons

\begin{tabular}{lrrrrrrrrrr}
\hline & \multicolumn{3}{c}{ Left-Cue Trial } & & \multicolumn{3}{c}{ Right-Cue Trial } & \\
\cline { 2 - 4 } & Enh & Sup & No-eff & & Enh & Sup & No-eff & & Total \\
\hline CLO (ima) & 13 & 0 & 6 & & 12 & 0 & 9 & 40 \\
CLO (ipa) & 6 & 0 & 3 & & 7 & 1 & 3 & 20 \\
YOH (ipa) & 1 & 12 & 10 & & 1 & 16 & 6 & 46 \\
Subtotal & 20 & 12 & 19 & & 20 & 17 & 18 & \\
Total & & 51 & & & 55 & & 106 \\
\hline
\end{tabular}

Abbreviations: Enh, enhancement; Sup, suppression; No-eff, no effect; CLO, clonidine; $\mathrm{YOH}$, yohimbine; ima, intramuscularly administered, ipa, iontophoretically applied.

ing sites of the delay-related neurons were reconstructed according to the traces of electrolytic lesions, or according to the coordinate readings between electrode penetrations and the reference site in those cases where the electrolytic lesion was difficult to identify.

\section{RESULTS}

A total of 82 electrode penetrations were made in the left prefrontal cortex (44 penetrations for Monkey No. 1 and 38 for Monkey No. 2). All of the electrode penetrations were done with an inserting angle of 40-45 degrees to sagittal plane and 0 degrees to coronal plane. A total of 106 neurons with delay-related activity were sampled from these penetrations and were successfully examined with the drugs. The 106 neurons showed an increase in activity selectively during the delay of leftcue ( $n=51$ neurons) or right-cue ( $n=55$ neurons) trials (Table 1).

Histological examination and re-construction of recording sites showed that 90 neurons were recorded from the principal sulcal area (40 neurons from the fundus, 14 from the ventral bank, and 36 from the dorsal bank of the principal sulcus; Walker's area 46), and 16 neurons from the cortical area dorsal to the principal sulcus (Walker's area 9). Figure 2 shows the distribution of the delay-related neurons in the prefrontal cortex.

As the delayed-response task used in the present study was not an oculomotor task, the eye position or eye movement was not controlled when the monkeys performed the task. Thus, it was possible that the monkeys could have developed a strategy to perform the task, i.e., the monkeys could have "cheated" by keeping their eyes fixated at a cued position (the left or right LED or button switch) during the delay. If this were true, the delay-related activity would represent the eye's fixation at a cued position instead of the working

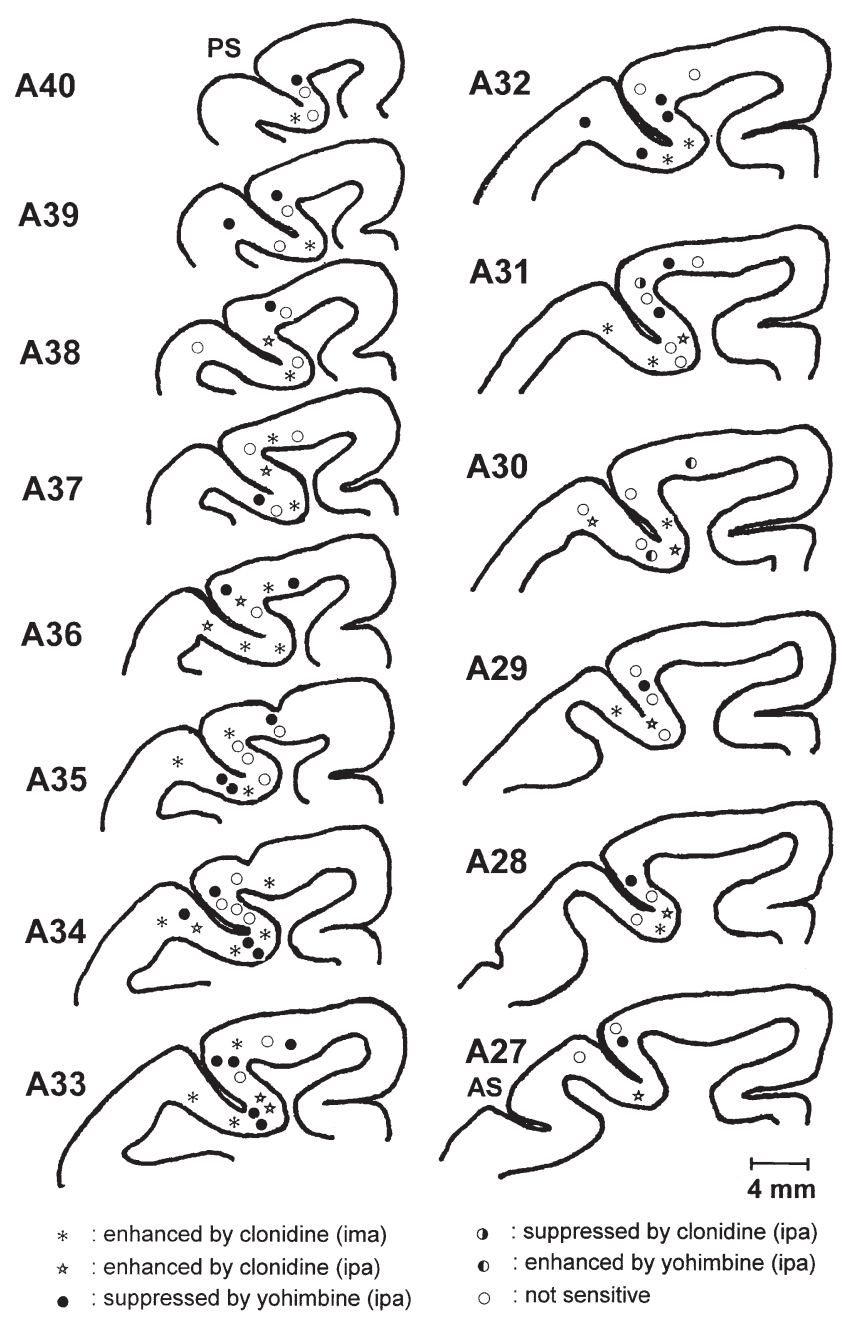

Figure 2. Distribution of the delay-related neurons $(n=$ 106) in the prefrontal cortex. Abbreviations: PS, principal sulcus; AS, arcuate sulcus; ima, intramuscularly administered; ipa, iontophoretically applied. For details see the text.

memory for the cued position. However, EOG monitoring of eye position demonstrated that this was not the case. Horizontal EOG data showed that the monkeys moved their eyes irregularly during the waiting and cue periods (i.e., searching for the cue signal), and generally fixated at the central LED instead of a cued LED during the delay (i.e., trying to detect the appearance of the choice signal). They directed their eyes to the cued LED only during the choice period (see Figure 3).

All of the 106 delay-related neurons sampled in the present study were not correlated in any manner with eye position or eye movement. Also, the activation during the delay can not be explained as a preparatory activity for eye movement or pressing movement toward a cued LED or button switch, because it did not show a gradually-increasing profile relative to these movements. Therefore, we argue that the delay-related activity selective for the left-cue or right-cue trial was a neu- 


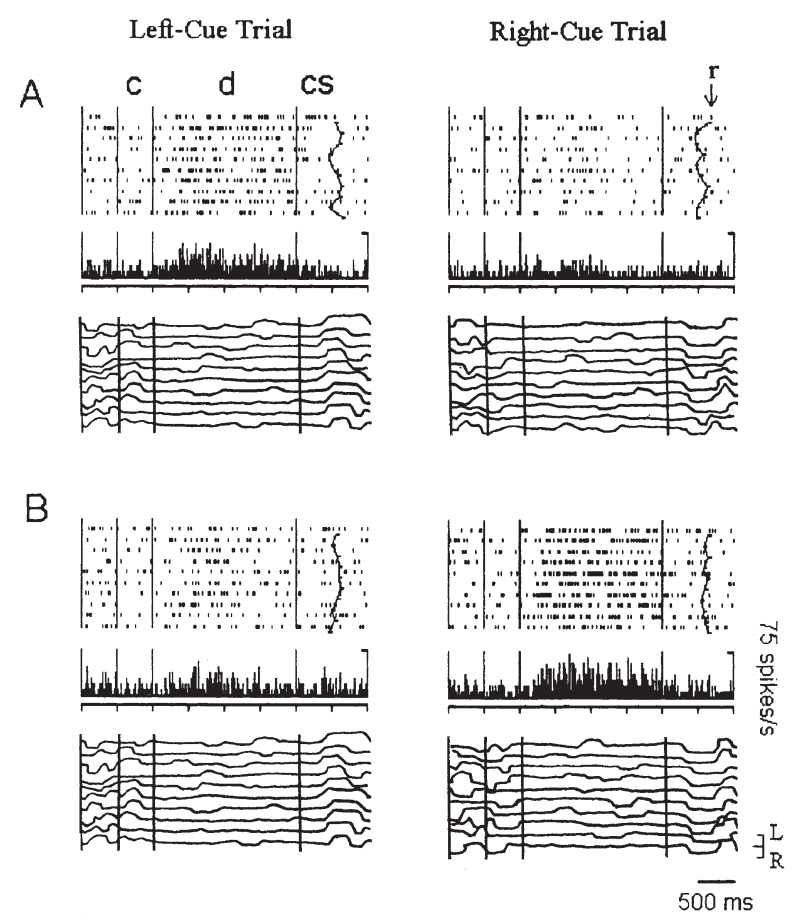

Figure 3. Delay-related activity and horizontal EOG data. Each panel includes a 10-trial raster display of neuronal activity (upper), a histogram display of the neuronal activity from the 10 trials (middle), and a 10-trial display of EOG (lower). (A) This neuron showed an increase in activity selectively during the delay of left-cue trials. EOG recordings showed that the monkey did not fixate its eyes at the left LED (or button switch) during the delay. (B) This neuron showed an increase in activity selectively during the delay of right-cue trials. EOG data showed that the monkey did not fixate its eyes at the right LED (or button switch) during the delay. The four vertical lines in each display represent the onsets of the lever-press, the cue, the delay, and the choice period, respectively. For the displays of neuronal activity and EOG in each panel, the upper-most trial was the 1st trial and the lower-most trial was the 10th trial. Bin width is $20 \mathrm{~m}$ for raster and histogram. Abbreviations: c, cue; d, delay; cs, choice signal; r, response (pressing button-switch); L, left; R, right.

ronal representation of the working memory coding the spatial position of a cued LED (button switch). The response of these cells was similar to the delay-related activity of PFC cells recorded by others (e.g., Funahashi et al. 1989).

Figure 3 shows the delay-related activities of two prefrontal cortical neurons and the horizontal EOGs. Neuron A showed an increase in activity during the delay of left-cue trials (A, left; the baseline activity was 10.3 spikes/sec and the delay-related activity 27.0 spikes $/$ sec; $p<.001$ for the delay-related activity vs. the baseline activity; Mann-Whitney $U$-test), but not during the delay of right-cue trials (A, right). Conversely, neu-
Table 2. Eye Movement and Delay-Related Activities of Neurons A and B Shown in Figure 3

\begin{tabular}{ccccc}
\hline & \multicolumn{4}{c}{$\begin{array}{c}\text { Number of Eye Movement } \\
\text { Deflections during the Delay }\end{array}$} \\
\cline { 2 - 6 } & $\mathbf{0}$ & $\mathbf{1}$ & $\mathbf{2}$ & $\mathbf{3}$ \\
\hline $\begin{array}{c}\text { Delay-related activity of } \\
\text { neuron A (spikes/sec) }\end{array}$ & 27.5 & 26.1 & 26.8 & 28.6 \\
$\begin{array}{c}\text { Delay-related activity of } \\
\text { neuron B (spikes/sec) }\end{array}$ & 32.4 & 33.1 & 31.7 & 32.0 \\
\hline
\end{tabular}

Each data is the average of delay-related activities form 4-8 trials (leftcue trials for neuron A and right-cue trials for neuron B) with delay of 2 sec before drug application. The delay-related activity did not change with the increase of the number of eye movement deflections (EMDs) occurring during the delay $(p>.05$ for the delay-related activity with 0 EMDs vs. that with 1, 2, or 3 EMDs; Mann-Whitney $U$-test).

ron $B$ showed an increase in activity during the delay of right-cue trials $(\mathrm{B}$, right; the baseline activity was 13.8 spikes/sec and the delay-related activity 33.5 spikes/ sec; $p<.001$ for the delay-related activity vs. the baseline activity), but not during the delay of left-cue trials (B, left). EOG data showed that, the monkey fixated at the central LED during the delay, or it made some eyemovement deflections but did not keep its eyes fixated at a cued position (see the EOG traces). For neuron A, of 116 eye-movement deflections occurring during the delay of 65 left-cue trials with correct behavioral response, $51.7 \%$ deflections were directed to the left and $48.3 \%$ to the right.

For neuron B, of 104 eye-movement deflections occurring during the delay of 70 right-cue trials with correct behavioral response, $53.8 \%$ deflections were directed to the left and $46.2 \%$ to the right. Thus, eyemovement deflections during the delay did not show preference to the direction of the cue (see the EOG traces). Moreover, the eye-movement deflections during the delay had no tuning effect on the delay-related activities of the two neurons. The delay-related activities did not change with the increase of the number of the eye-movement deflections (see Table 2). Therefore, the sustained delay-related activities were not related to monkey's eye position or movement, but to working memory for a cued spatial position. The delay-related activity of neuron A was sensitive to systemically administered clonidine and that of neuron B to iontophoretically applied yohimbine.

Table 1 summarizes the effects of clonidine and yohimbine on the 106 delay-related neurons. Mann-Whitney's $U$-test was used for each neuron to test if the baseline and delay-related activities were sensitive to drug treatment. Intramuscularly injected clonidine significantly enhanced delay-related activity in 25 of 40 examined neurons and had no effect in the remaining 15 neurons (Table 1). In the 25 neurons enhanced by clonidine, 
the mean value (spikes/sec) of delay-related activity was increased by $32.5 \% \pm 14.5 \%(p<.01$, paired $t$-test), while that of baseline activity by only $4.8 \% \pm 12.6 \%(p>$ $.05)$. In 21 of the 25 neurons ( $84 \%)$, the facilitatory effect of clonidine was antagonized by iontophoretically applied yohimbine, indicating that the effect of clonidine was mediated by alpha-2 adrenoceptors in the prefrontal cortex. The facilitatory effect of clonidine appeared 3.0-8.0 min after injection, and we were not able to determine how long the effect could last because the activity of an examined neuron disappeared after it had been recorded for 0.5-1.5 hrs. Thus, the effect of systemically administered clonidine was mainly excitatory on delay-related activity ( 25 enhanced neurons vs. 0 inhibited neurons), and was mediated by alpha- 2 adrenoceptors in the prefrontal cortex. As the monkeys performed the task with great accuracy before drug administration $(94.2 \% \pm 2.4 \%$ correct), there was little room for clonidine to improve performance (after clonidine: $96.5 \% \pm$ $1.6 \%$ correct; mean $\pm \mathrm{SD} ; n=25$ daily sessions).

Figure 4 shows the activity of a delay-related neuron that was recorded from the fundus of the principal sulcus and was sensitive to intramuscularly injected clonidine. This neuron increased its discharge rate during the delay of the left-cue trials (A, left; the baseline activity was 9.2 spikes/sec and the delay-related activity was 23.6 spikes /sec; $p<.001$ for the delay-related activity vs. the baseline activity, Mann-Whitney's $U$-test), but not during the delay of the right-cue trials (A, right; the baseline activity was 8.2 spikes/sec and the activity during the delay was 11.4 spikes $/ \mathrm{sec} ; p>.05$ for the activity during the delay vs. the baseline activity). Intramuscularly injected clonidine significantly enhanced the delay-related activity ( $\mathrm{B}$, left; the delay-related activity was 34.6 spikes/sec; $p<.01$ as compared with A, Mann-Whitney's $U$-test). The facilitatory effect of clonidine was blocked by iontophoretically applied yohimbine with $50 \mathrm{nA}$ current $(\mathrm{C}$, left; the delay-related activity was 22.9 spikes/sec; $p>.05$ vs. A and $p<.01$ vs. B), and recovered after the termination of yohimbine iontophoresis $(\mathrm{D}$, left; the delay-related activity was 37.3 spikes /sec; $p<.01$ vs. A and C, and $p>.05$ vs. B). The baseline activity in either the left-cue or right-cue trials was not changed during the drug treatments. For the left-cue trials the baseline activity was 10.6 spikes/sec in $B, 12.8$ spikes/sec in C, and 12.0 spikes/sec in D ( $p>$ .05 for $B$ vs. A, C vs. B, and D vs. C) . For the right-cue trials the baseline activity was 12.0 spikes / sec in B, 10.4 spikes/sec in C, and 10.8 spikes/sec in D ( $p>.05$ for B vs. A, C vs. B, and D vs. C). The activity during the delay of the right-cue trials was also unchanged during the drug treatments (11.6 spikes/sec in B, 10.0 spikes/s in $\mathrm{C}$, and 12.6 spikes/sec in $\mathrm{D} ; p>.05$ for B vs. A, C vs. B, and D vs. C).

Iontophoretically applied clonidine significantly enhanced delay-related activity in 13 of 20 examined neu-
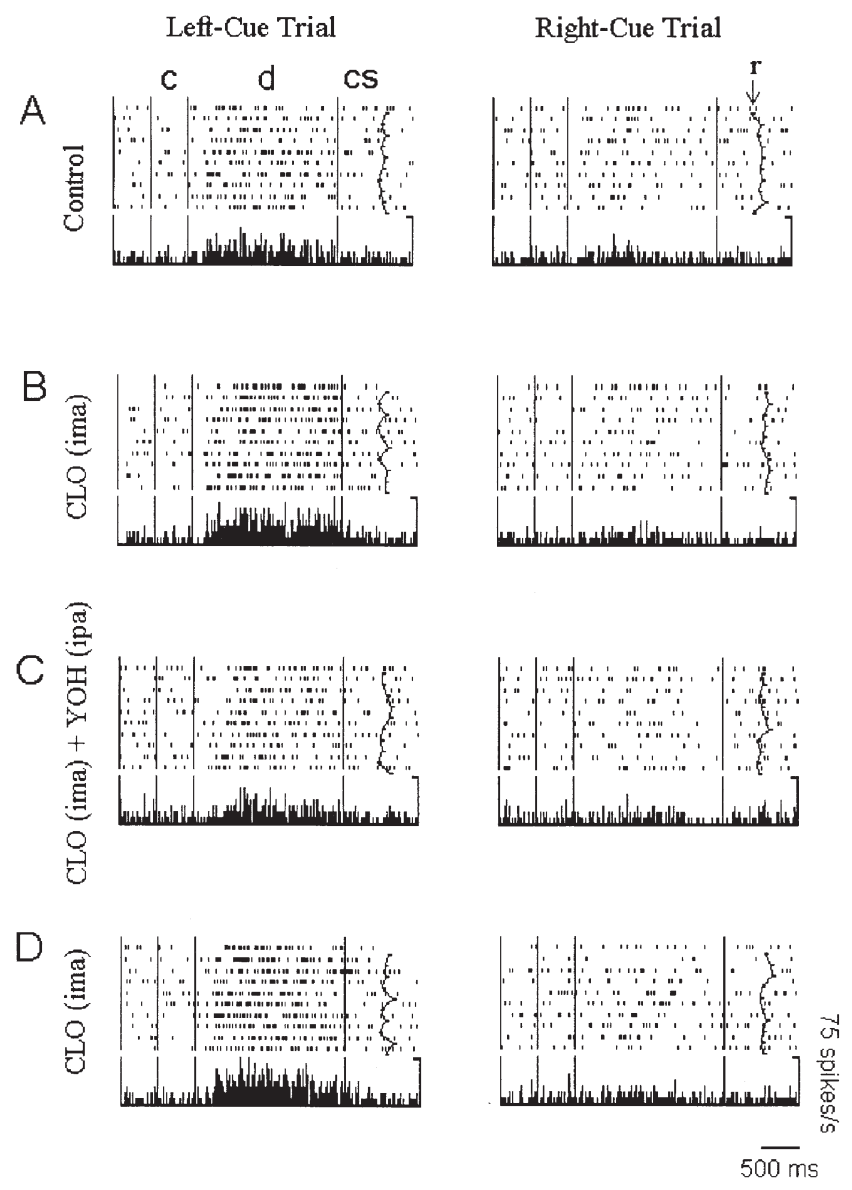

Figure 4. Facilitatory effect of intramuscularly administered clonidine $(0.04 \mathrm{mg} / \mathrm{kg})$ on delay-related activity. The facilitatory effect was antagonized by iontophoretically applied yohimbine (25 nA iontophoresis current). Each panel includes a 10-trial raster display of neuronal activity (upper) and a histogram display of the neuronal activity from the 10 trials (lower). Abbreviations: ima, intramuscularly administered; ipa, iontophoretically applied. Other formats as in Figure 3. For details see the text.

rons, suppressed it in one neuron and had no effect in the remaining six neurons (Table 1). The facilitatory effect of clonidine was blocked by similarly applied yohimbine. In the 13 neurons enhanced by clonidine, the mean value (spikes/sec) of delay-related activity was increased by $38.2 \% \pm 18.6 \%$ (mean \pm S.D., $p<.01$, paired $t$-test), while that of baseline activity by $7.2 \% \pm$ $14.5 \%(p>.05)$. The facilitatory effect of clonidine appeared 1.0-4.0 min after onset of iontophoresis current and disappeared within 2.0-4.0 min of stopping the iontophoresis current. Thus, the effect of iontophoretically applied clonidine was mainly excitatory on delayrelated activity (13 enhanced neurons vs. one inhibited neuron), an effect similar to that of intramuscularly injected clonidine (Figure not shown). The facilitatory effect of iontophoretically applied clonidine on the work- 
ing memory related activity was relatively stronger than that of systemically administered clonidine $(38.2 \% \pm$ $18.6 \%$ enhancement by iontophoretically-applied clonidine vs. $32.5 \% \pm 14.5 \%$ enhancement by systemicallyadministered clonidine), but the difference is not statistically significant $(p>.05$, either Mann-Whitney $U$-test or unpaired $t$-test).

Iontophoretically applied yohimbine significantly enhanced delay-related activity in 2 of 46 examined neurons, suppressed it in 28 neurons, and had no effect in the remaining 16 neurons. In the 28 neurons suppressed by yohimbine, the mean value (spikes/sec) of delay-related activity was decreased by $34.0 \% \pm 17.8 \%$ (mean $\pm \mathrm{SD}, p<.01$, paired $t$-test), while baseline activity was decreased by $5.5 \% \pm 8.2 \%(p>.05)$. The inhibitory effect of yohimbine appeared 1.0-4.0 minutes after onset of iontophoresis current and disappeared within 2.0-4.0 minutes of stopping iontophoresis current. Thus, the effect of iontophoretically applied yohimbine was mainly inhibitory on delay-related activity (28 inhibited neurons vs. two enhanced neurons).

Figure 5 shows the activity of a delay-related neuron which was recorded from the upper bank of the principal sulcus and was sensitive to iontophoretically applied yohimbine. This neuron increased its discharge rate during the delay of the right-cue trials (A, right; the baseline activity was 13.2 spikes/sec and the delayrelated activity was 31.7 spikes $/ \mathrm{sec} ; p<.001$ for the delay-related activity vs. the baseline activity, MannWhitney's $U$-test), but not during the delay of the leftcue trials (A, left; the baseline activity was 15.8 spikes/ sec and the activity during the delay was 18.8 spikes/ sec; $p>.05$ for the activity during the delay vs. the baseline activity). Iontophoretically applied yohimbine (25 nA current) significantly suppressed the delay-related activity $(B$, right; the delay-related activity was 25.0 spikes/sec; $p<.05$ vs. A, Mann-Whitney's $U$-test). The suppression became more evident when the iontophoresis current was $50 \mathrm{nA}(\mathrm{C}$, right; the delay-related activity was 20.0 spikes/sec; $p<.01$ vs. A and $p<.05$ vs. B). The delay-related activity recovered after the termination of iontophoresis current $(\mathrm{D}$, right; the delayrelated activity was 31.5 spikes $/ \mathrm{sec} ; p>.05$ vs. A and $p<.01$ vs. C). The baseline activity in either the rightcue or left-cue trials was not changed during the drug treatments. For the right-cue trials the baseline activity was 12.4 spikes/sec in B, 16.6 spikes/sec in C, and 14.8 spikes /sec in D ( $p>.05$ for B vs. A, C vs. B, and D vs. C). For the left-cue trials the baseline activity was 15.2 spikes/sec in $B, 17.6$ spikes/sec in $C$, and 17.2 spikes/sec in D ( $p>.05$ for B vs. A, C vs. B, and D vs. C). The activity during the delay of the left-cue trials was also unchanged during the drug treatments (18.2 spikes/sec in B, 17.4 spikes/sec in C and 17.2 spikes/ sec in $\mathrm{D} ; p>.05$ for $\mathrm{B}$ vs. $\mathrm{A}, \mathrm{C}$ vs. $\mathrm{B}$, and $\mathrm{D}$ vs. $\mathrm{C})$. The raw spike traces of this neuron before and during yo-
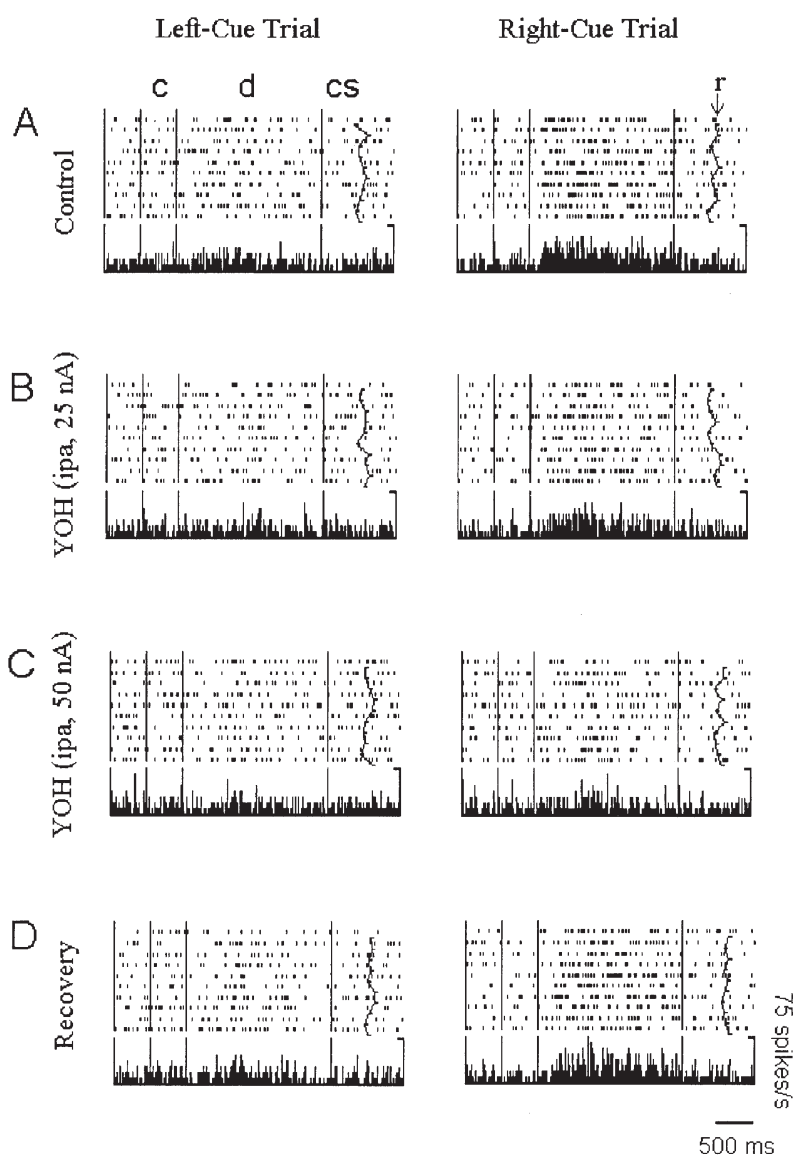

Figure 5. Inhibitory effect of iontophoretically applied yohimbine on delay-related activity. Each panel includes a 10-trial raster display of neuronal activity (upper) and a histogram display of the neuronal activity from the 10 trials (lower). Abbreviations: ipa, iontophoretically applied. Other formats as in Figure 3. For details see the text.

himbine iontophoresis are presented in Figure 6. The spike shape and amplitude was not changed during the iontophoresis.

\section{DISCUSSION}

The main findings of the present study were that: 1) systemically administered clonidine enhanced PFC neuronal activity related to spatial working memory and this effect was mediated by alpha- 2 adrenoceptors in the PFC; and 2) iontophoretically applied clonidine enhanced PFC neuronal activity related to spatial working memory, while yohimbine suppressed it.

Spatial working memory has been documented to be sensitive to the manipulation of alpha-2 adrenoceptors (Arnsten and Goldman-Rakic 1985; Arnsten et al. 1988, 1996; Li and Mei 1994a; Franowicz and Arnsten 1999). Arnsten and coworkers reported that systemi- 
A

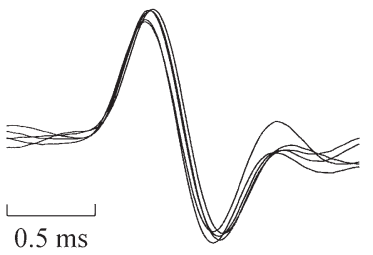

B

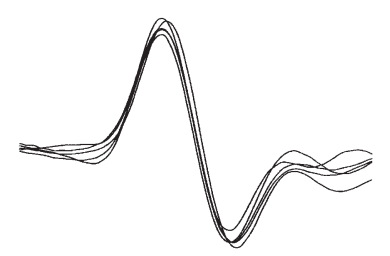

Figure 6. Raw spike traces of the neuron shown in Figure 5. (A) and (B) represent the spike traces before and during yohimbine iontophoresis (50 nA current), respectively. The spike shape and amplitude were not altered when yohimbine was applied iontophoretically.

cally-administered clonidine (or guanfacine) improved the spatial working memory performance of aged monkeys with naturally-occurring NE depletion or young monkeys with experimentally-induced NE depletion and suggested that the beneficial effect of alpha- 2 agonists was mediated by postsynaptic alpha- 2 adrenoceptors in the PFC (Arnsten and Goldman-Rakic 1985; Arnsten et al. 1988).

The dose of systemically-administered clonidine used in the current study $(0.04 \mathrm{mg} / \mathrm{kg})$ is within the dose range $(0.02-0.1 \mathrm{mg} / \mathrm{kg})$ shown to enhance delayed response performance in normal young adult monkeys (Franowicz and Arnsten 1999), indicating that enhanced performance on a spatial working memory task is associated with enhanced delay-related activity in the PFC. Furthermore, we reported previously that locally infused yohimbine into the PFC impaired spatial working memory performance of young monkeys ( $\mathrm{Li}$ and Mei 1994a).

The present study showed that iontophoretically applied yohimbine had an inhibitory effect, whereas clonidine had a facilitatory effect on PFC neuronal activity related to spatial working memory. These results strongly support the studies by Arnsten and coworkers utilizing systemic administration of alpha-2 agonists and antagonists (Arnsten and Goldman-Rakic 1985; Arnsten et al. 1988) and by ourselves utilizing local infusion of an alpha-2 antagonist (Li and Mei 1994a), and provide neurophysiological evidence for the involvement of alpha-2 adrenoceptors in the PFC in the neural mechanisms underlying spatial working memory. In particular, the findings with yohimbine indicate that endogenous NE stimulation of alpha-2 adrenoceptors in the PFC has an important influence on working memory function. These results are consistent with recordings from NE locus coeruleus neurons, which demonstrate that NE cells fire to cognitively meaningful stimuli (Aston-Jones et al. 1994; Usher et al. 1999).

The delayed-response task used in the present study had only two cue positions, and all of the PFC neurons examined showed an increase in activity during the delay of either left-cue or right-cue trials. Thus, the spatial selectivity of each PFC neuron was "100\%" to left-cue or right-cue position, either before or during administration of clonidine. Therefore, the information concerning the modulation by clonidine of the spatial selectivity of the PFC neurons was not available. Enhanced delay-related activity does not necessarily mean the increase of the ability of the PFC neurons to convey spatial information. However, as it has been demonstrated that systemically administered clonidine can improves monkey's performance on spatial working memory task (Arnsten and Goldman-Rakic 1985, Franowicz and Arnsten 1999), we argue that the increase by clonidine of delay-related activity is beneficial to spatial working memory, instead of interfering it by adding "noise".

Behavioral, pharmacological, and electrophysiological studies in nonhuman primates have shown that stimulation of alpha-2 adrenoceptors produces a beneficial influence on PFC cognitive functions such as attention regulation, response inhibition and working memory (Arnsten et al. 1996; Li and Kubota 1998). Most recently, Li and Kubota (1998) reported that iontophoretically applied clonidine enhanced PFC neuronal activity related to a visual discrimination task with go and no-go performance, a task which required the monkeys to initiate or inhibit a motor response according to visual guidance. Alpha-2 agonists, such as clonidine and guanfacine, have been developed and used experimentally or clinically for the treatment of human psychiatric disorders such as schizophrenia (Fields et al. 1988), Korsakoff's syndrome (Mair and McEntee 1986), and especially Attention Deficit Hyperactivity Disorder (Hunt et al. 1985, 1995; Chappell et al. 1995; Horrigan and Barnhill 1995). Patients with Attention Deficit Hyperactivity Disorder show prominent PFC cognitive deficits including poor attention regulation (attention deficit), inability to inhibit motor activity (hyperactivity), and poor working memory (Barkley et al. 1992). The present study shows that systemically administered clonidine can facilitate PFC neuronal activity related to spatial working memory through actions at alpha-2 adrenoceptors in the PFC. Our data provide a neurophysiological explanation for the beneficial influence of systemically administered alpha- 2 agonists on PFC cognitive functions (such as working memory) in nonhuman primates or human patients with PFC disorders. Systemically administered clonidine could act at alpha-2 adrenoceptors in the whole central nervous system. Although the facilitatory effect of clonidine could be blocked by yohimbine applied iontophoretically into the PFC, it can not be excluded completely that systemically administered clonidine could modulate working memory function of the PFC through actions at alpha-2 adrenoceptors in other brain structures such as the locus coeruleus. 
Sawaguchi and coworkers reported that D1 dopaminergic receptors in the PFC are also important for spatial working memory (Sawaguchi et al 1990; Sawaguchi and Goldman-Rakic 1994). It remains to be studied how alpha-2 adrenergic and D1 dopaminergic receptors coordinate in their modulation of delay-related activity. However, the present report demonstrates that noradrenergic modulation through alpha-2 adrenergic receptors has a powerful influence on the cellular mechanisms underlying spatial working memory.

\section{SUMMARY}

The present study has demonstrated that: 1) systemically administered alpha-2 adrenergic agonist clonidine enhanced prefrontal cortical neuronal activity related to spatial working memory (SWM) and that this effect was mediated by alpha-2 adrenoceptors in the prefrontal cortex; and 2) iontophoretically applied clonidine enhanced SWM-related prefrontal cortical neuronal activity, whereas similarly applied alpha-2 antagonist yohimbine suppressed it. The present study has provided neurophysiological evidence that alpha-2 adrenoceptors in the prefrontal cortex are involved in the neural mechanisms underlying spatial working memory and that systemically administered alpha- 2 agonist could act at prefrontal cortical alpha-2 adrenoceptors to exert a facilitatory effect on SWM-related neuronal activity.

\section{ACKNOWLEDGMENTS}

The authors thank Ms. K.-J. Xu for her technical assistance during this study, Drs. A.F.T. Arnsten and M. Inase for their helpful comments on the manuscript. This work was supported by grants from the National Natural Science Foundation of China ( No. 39000032 and No. 39630140), the Chinese Academy of Sciences (No. STZ-2-05), and the Ministry of Science and Technology of China (Climbing Project 95-YU-1812).

\section{REFERENCES}

Arnsten AFT, Goldman-Rakic PS (1985): Alpha-2-adrenergic mechanisms in prefrontal cortex associated with cognitive decline in aged nonhuman primates. Science 230:1273-1276

Arnsten AFT, Cai JX, Goldman-Rakic PS (1988): The alpha-2 adrenergic agonist guanfacine improves memory in aged monkeys without sedative or hypotensive side effects: Evidence for alpha-2 receptor subtypes. J Neurosci 8:4287-4298

Arnsten AF, Leslie FM (1991): Behavioral and receptor binding analysis of the alpha-2 adrenergic agonist, UK-14304 (5 bromo-6 [2-imidazoline-2-yl amino] quinoxaline): Evidence for cognitive enhancement at an alpha-2 adrenoceptor subtype. Neuropharmacology 30:1279-1289
Arnsten AFT, Steere JC, Hunt RD (1996): The contribution of alpha-2 noradrenergic mechanisms to prefrontal cortical cognitive function. Arch Gen Psychiatry 53:448-455

Arnsten AFT, van Dyck H (1997): Monoamine and acetylcholine influences on higher cognitive functions in nonhuman primates: Relevance to the treatment of Alzheimer's disease. In Brioni JD, Decker MW (eds), Pharmacological Treatment of Alzheimer's Disease: Molecular and Neurobiological Foundations. New York, John Wiley \& Sons, pp 63-86

Aston-Jones G, Rajkowski J, Kubiak P, Alexinsky T (1994): Locus coeruleus neurons in the monkey are selectively activated by attended cues in a vigilance task. J Neurosci 14:4467-4480

Barkley RA, Grodzinsky G, DuPaul GJ (1992): Frontal lobe functions in Attention Deficit Disorder with and without Hyperactivity: A review and research report. J Abnormal Child Psychology 20:163-188

Brozoski TJ, Brown RM, Rosvold HE, Goldman, PS (1979): Cognitive deficit caused by regional depletion of dopamine in prefrontal cortex of rhesus monkey. Science 215:929-932

Cai JX, Ma Y, Xu L, Hu X (1993): Reserpine impairs spatial working memory performance in monkeys: Reversal by the alpha-2 adrenergic agonist clonidine. Brain Res 614:191-196

Chappell PB, Riddle MA, Scahill L, Lynch K, Schultz R, Arnsten AFT, Leckman JF, Cohen DL (1995): Guanfacine treatment of comorbid attention deficit hyperactivity disorder and Tourette's Syndrome: Preliminary clinical experience. J Am Acad Child Adol Psychiatry 34:11401146

di Pellegrino G, Wise SP (1993): Visuospatial versus visuomotor activity in the premotor and prefrontal cortex of a primate. J Neurosci 13:1227-1243

Fields RB, Van Kammen DP, Peters JL, Rosen J, Van Kammen WB, Nugent A, Stipetics S, Linnoila M (1988): Clonidine improves memory function in schizophrenia independently from change in psychosis. Schizophr Res 1:417-423

Franowicz JCS, Arnsten AFT (1999): Treatment with the noradrenergic alpha-2 agonist clonidine, but not diazepam, improves spatial working memory in normal young rhesus monkeys. Neuropsychopharmacology 25:611-621

Funahashi S, Bruce CJ, Goldman-Rakic PS (1989): Mnemonic coding of visual space in the primate dorsolateral prefrontal cortex. J Neurophysiol 61:331-349

Funahashi S, Kubota K (1994): Working memory and prefrontal cortex. Neurosci Res 21:1-11

Fuster JM, Bauer RH, Jervey JP (1982): Cellular discharge in the dorsolateral prefrontal cortex of the monkey in cognitive tasks. Exp Neurol 77:679-694

Goldman-Rakic PS (1987): Circuitry of the primate prefrontal cortex and the regulation of behavior by representational memory. In F. Plum (ed), Handbook of Physiology, The Nervous System, Higher Functions of the Brain, Vol V, Sect I. Bethesda, MD, American Physiology Society, pp 373-417

Goldman-Rakic PS (1996): Regional and cellular fraction- 
ation of working memory. Proc Natl Acad Sci USA 93:13473-13480

Horrigan JP, Barnhill LJ (1995): Guanfacine for treatment of Attention Deficit Hyperactivity Disorder in boys. J Child Adol Psychopharmacol 5:215-223

Hunt RD, Minderaa RB, Cohen DJ (1985): Clonidine benefits children with attention deficit disorder and hyperactivity: report of a double-blind placebo-crossover therapeutic trial. J Am Acad Child Psychiatry 24:617-629

Hunt RD, Arnsten AFT, Asbell MD (1995): An open trial of guanfacine in the treatment of attention deficit hyperactivity disorder. J Am Acad Child Adolesc Psychiatry 34:50-54

Li B-M, Mei Z-T, Kubota K (1990): Multibarreled glasscoated tungsten microelectrode for both neuronal activity recording and iontophoresis in monkeys. Neurosci Res 8:214-219

Li B-M, Mei Z-T (1994a): Delayed-response deficit induced by local injection of the alpha- 2 adrenergic antagonist yohimbine into the dorsolateral prefrontal cortex in young adult monkeys. Behav Neural Biol 62:134-139

Li B-M, Mei Z-T (1994b): Alpha-2 adrenergic modulation of prefrontal neuronal activity related to working memory in monkeys. Abstract of the 3rd Congress of Federation of Asian and Oceanian Physiological Societies, S9-3, p 96

Li B-M, Kubota K (1998): Alpha-2 adrenergic modulation of prefrontal cortical neuronal activity related to a visual discrimination task with GO and NO-GO performances in monkeys. Neurosci Res 31:83-95

Mair RG, McEntee WJ (1986): Cognitive enhancement in
Korsakoff's psychosis by clonidine: a comparison with 1-dopa and ephedrine. Psychopharmacology 88:374-380

Miller EK, Erickson CA, Desimone R (1996): Neural mechanisms of visual working memory in prefrontal cortex of the macaque. J Neurosci 16:5154-5167

Rama P, Linnankoski I, Tanila H, Pertovaara A, Carlson S (1996) Medetomidine, atipamezole, and guanfacine in delayed response performance of aged monkeys. Pharmacol Biochem Behav 54:1-7

Rao SC, Rainer G, Miller EK (1997): Integration of what and where in the primate prefrontal cortex. Science 276:821824

Sawaguchi T, Matsumura M, Kubota K (1990): Effects of dopamine antagonists on neuronal activity related to a delayed response task in monkey prefrontal cortex. J Neurophysiol 63:1401-1412

Sawaguchi T, Goldman-Rakic PS (1994): The role of D1dopamine receptor in working memory: Local injections of dopamine antagonists into the prefrontal cortex of rhesus monkeys performing an oculomotor delayedresponse task. J Neurophysiol 71:515-528

Tanila H, Rama P, Carlson S (1996): The effects of prefrontal intracortical microinjections of an alpha-2 agonist, alpha-2 antagonist and lidocaine on the delayed alternation performance of aged rats. Brain Res Bull 40:117119

Usher M, Cohen JD, Servan-Schreiber D, Rajkowski J, AstonJones G (1999): The role of locus coeruleus in the regulation of cognitive performance. Science 283:549-554

Wilson FA, O'Scalaidhe SP, Goldman-Rakic PS (1993): Dissociation of object and spatial processing domains in primate prefrontal cortex. Science 260:1955-1958 